\title{
Encapsulation of multi-enzymes on waste clay material: preparation, characterization and application for tapioca starch hydrolysis
}

\begin{abstract}
Alpha-amylase, glucoamylase and cellulase were immobilized onto calcium alginate-waste clay (alg-WC) beads by using encapsulation technique. The clay was obtained from palm oil mill clay bath processing waste that was collected from FELDA Serting Hilir Palm Oil Mill, Malaysia. The aim of this study is to characterize and investigate the performance of waste clay as a supporting material for the tapioca starch hydrolysis. The encapsulated enzymes were characterized by using analytical equipment such as Fourier Transform Infrared Spectroscopy (FTIR) and Field Emission Scanning Electron Microscopy (FESEM). The FTIR results showed the interaction of functional groups between alginate and waste clay in the alg-WC beads. The alginate beads characterized at peaks $1615 \mathrm{~cm}-1,1417 \mathrm{~cm}-1$ and 1027 $\mathrm{cm}-1$, and peaks $1396 \mathrm{~cm}-1,873 \mathrm{~cm}-1$ and $712 \mathrm{~cm}-1$ characterized the waste clay. The FESEM analysis also revealed that the different surface morphology of alginate beads and alg-WC beads. It also showed that the enzymes were successfully encapsulated within the alg-WC beads. The activity of the immobilized enzymes was studied at different clay concentrations. The highest immobilization yield was obtained at $2 \%(\mathrm{w} / \mathrm{v})$ of clay concentration and the highest enzyme loading was $98.29 \%$. The immobilized enzymes also retained $33 \%$ of its activity after seven usage cycle with 1 hour of each reaction time.
\end{abstract}

Keyword: Enzyme encapsulation; FESEM; FTIR; Immobilization yield; Waste clay 